\title{
Bistatic High Frequency Radar Ocean Surface Cross Section for an FMCW Source with an Antenna on a Floating Platform
}

\author{
Yue Ma, Weimin Huang, and Eric W. Gill \\ Faculty of Engineering and Applied Science, Memorial University of Newfoundland, St. John's, NL, Canada A1B 3X5 \\ Correspondence should be addressed to Yue Ma; ym4428@mun.ca
}

Received 5 February 2016; Revised 13 May 2016; Accepted 29 May 2016

Academic Editor: Atsushi Mase

Copyright (C) 2016 Yue Ma et al. This is an open access article distributed under the Creative Commons Attribution License, which permits unrestricted use, distribution, and reproduction in any medium, provided the original work is properly cited.

\begin{abstract}
The first- and second-order bistatic high frequency radar cross sections of the ocean surface with an antenna on a floating platform are derived for a frequency-modulated continuous wave (FMCW) source. Based on previous work, the derivation begins with the general bistatic electric field in the frequency domain for the case of a floating antenna. Demodulation and range transformation are used to obtain the range information, distinguishing the process from that used for a pulsed radar. After Fourier-transforming the autocorrelation and comparing the result with the radar range equation, the radar cross sections are derived. The new first- and second-order antenna-motion-incorporated bistatic radar cross section models for an FMCW source are simulated and compared with those for a pulsed source. Results show that, for the same radar operating parameters, the first-order radar cross section for the FMCW waveform is a little lower than that for a pulsed source. The second-order radar cross section for the FMCW waveform reduces to that for the pulsed waveform when the scattering patch limit approaches infinity. The effect of platform motion on the radar cross sections for an FMCW waveform is investigated for a variety of sea states and operating frequencies and, in general, is found to be similar to that for a pulsed waveform.
\end{abstract}

\section{Introduction}

The derivation of high frequency radar ocean surface cross sections has been studied for over four decades. The firstorder high frequency radar scatter cross section was developed and analysed in [1]. Later, Walsh and Gill [2] analysed the scattering of high frequency electromagnetic radiation of the ocean surface for a pulse radar. Then, Gill and Walsh [3] developed the bistatic radar cross section of the ocean surface. Following these analyses, the work was extended to the high frequency monostatic radar cross sections for an antenna on a floating platform $[4,5]$.

All of the models mentioned above were developed specifically for pulsed radar. However, there are inherent disadvantages to using pulsed radar systems. For example, the detectable range capability is determined by the average transmitted power. In a pulsed radar system, both the range resolution and the average transmitted power are dependent on the pulse width. Narrower pulses, bringing better range resolution, require large peak powers to be useful at long range. Compared to this, FMCW radar systems are able to achieve satisfactory range resolution and long range with moderate peak power due to a $100 \%$ duty cycle. Thus, in recent years, FMCW radars have been widely used in ocean remote sensing applications.

A good summary of the digital processing of an FMCW signal for radar systems has been reported by Barrick [6]. Two processing techniques were analysed and compared. Based on that work, the design of a frequency-modulated interrupted continuous wave radar was described and implemented in [7]. Then, techniques for range and unambiguous velocity measurement for an FMCW radar were outlined in [8]. More recently, Walsh et al. [9] developed the first- and secondorder monostatic radar ocean surface cross sections for an FMCW waveform. Also, the first-order FMCW radar cross section model for mixed-path ionosphere-ocean propagation has been established and simulated in [10].

In this paper, the first- and second-order bistatic radar ocean surface cross sections for an antenna on a floating platform and incorporating an FMCW source are presented. 


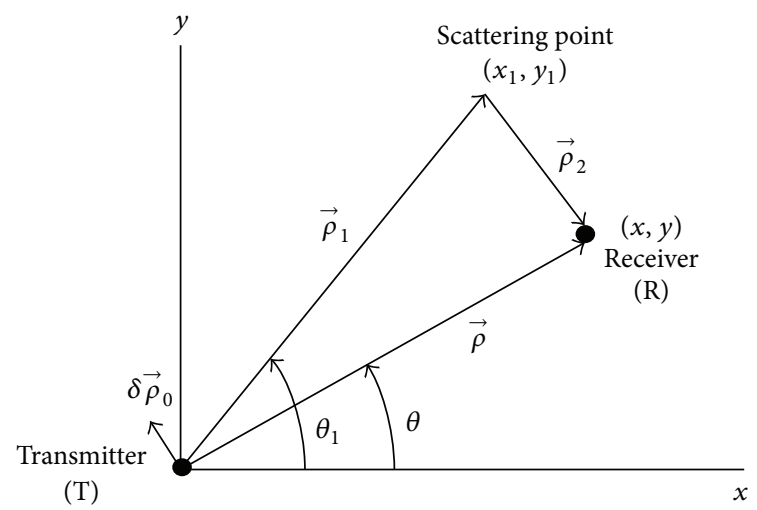

(a)

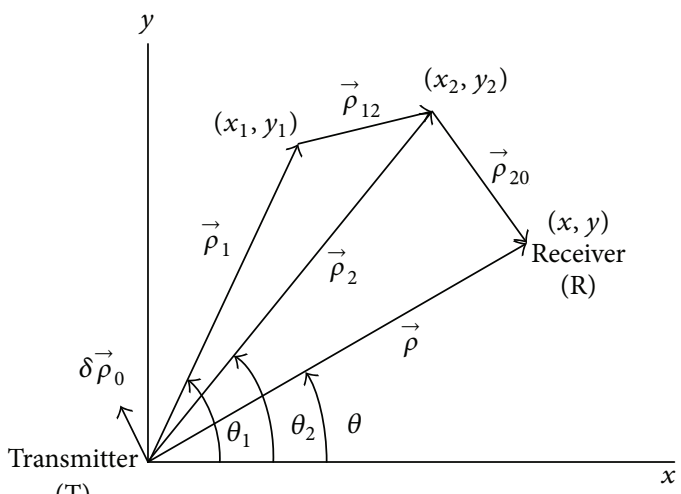

(b)

FIGURE 1: General (a) first-order and (b) second-order bistatic scatter geometry with antenna motion.

In Section 2, the derivation process of the first- and secondorder received electric field is reviewed. Then, a method similar to that in [3] is used to obtain the first- and secondorder radar cross section in Section 3. Section 4 contains model simulations and comparisons with the pulsed waveform. Section 5 provides conclusions.

\section{Radar Received Field Equations}

2.1. General First- and Second-Order Electric Field Equation. By using a small displacement vector, $\delta \vec{\rho}_{0}=\left(\delta \rho_{0}, \theta_{0}\right)$, to represent the sway motion of the platform (see Figure 1) and adding this small displacement in the source term, the first-order bistatic scattered field for the case of a floating transmitter and a fixed receiver appearing in [11] may be written for a vertical dipole source as

$$
\begin{aligned}
\left(E_{n}\right)_{1}= & \frac{k C_{0}}{(2 \pi)^{3 / 2}} \sum_{\vec{K}} P_{\vec{K}} \sqrt{K} e^{j(\rho K / 2) \cos \left(\theta_{K}-\theta\right)} \\
& \cdot \int_{\rho / 2}^{\infty} \frac{F\left(\rho_{1}\right) F\left(\rho_{2}\right)}{\sqrt{\rho_{s}\left[\rho_{s}^{2}-(\rho / 2)^{2}\right]}} e^{-j(\pi / 4)} \sqrt{\cos \phi} \\
& \cdot e^{j k \delta \rho_{0}\left[\cos \phi \cos \left(\theta_{K}-\theta_{0}\right)+\sin \phi \sin \left(\theta_{K}-\theta_{0}\right)\right]} \\
& \cdot e^{j \rho_{s}[K \cos \phi-2 k]} d \rho_{s} .
\end{aligned}
$$

Here, $C_{0}=I \Delta l k^{2} / j \omega \varepsilon_{0}$ is the dipole constant, with $I$ being the current on the dipole of length $\Delta l$. $\omega$ and $k$ are the radian frequency and wavenumber of the dipole current, respectively, in a space with permittivity of $\varepsilon_{0} . P_{\vec{K}}$ represents the Fourier coefficient of a surface component whose wave vector has magnitude $K$ and direction $\theta_{K}$ (i.e., $\vec{K}=\left(K, \theta_{K}\right)$ ). With reference to Figure $1(\mathrm{a}), \vec{\rho}=(\rho, \theta)$ is a distance vector, pointing from the transmitter to the receiver without incorporating the platform motion. $\rho_{s}=\left(\rho_{1}+\rho_{2}\right) / 2$ and $\phi$ is the bistatic angle. $F$ represents the Sommerfeld attenuation function.
The second-order bistatic received electric field corresponding to the first-order found in (1) appears in [12] as

$$
\begin{aligned}
\left(E_{n}\right)_{2}= & \frac{-k C_{0}}{(2 \pi)^{3 / 2}} \sum_{\vec{K}_{1}} \sum_{\vec{K}_{2}} \frac{P_{\vec{K}_{1}} P_{\vec{K}_{2}}}{\sqrt{K}} e^{j(\rho K / 2) \cos \left(\theta_{K}-\theta\right)} \\
& \cdot \int_{\rho / 2}^{\infty} \frac{(-k \chi) F\left(\rho_{2}\right) F\left(\rho_{20}\right)}{\sqrt{\rho_{s}\left[\rho_{s}^{2}-(\rho / 2)^{2}\right]}} \frac{e^{-j(\pi / 4)}}{\sqrt{\cos \phi}} \\
& \cdot e^{j k \delta \rho_{0}\left[\cos \phi \cos \left(\theta_{K}-\theta_{0}\right)+\sin \phi \sin \left(\theta_{K}-\theta_{0}\right)\right]} \\
& \cdot e^{j \rho_{s}[K \cos \phi-2 k]} d \rho_{s} .
\end{aligned}
$$

This expression accounts for the electric field arising due to the transmitted signal being scattered twice by the rough surface. $\vec{K}_{1}$ and $\vec{K}_{2}$ are the first and the second scattering wave vectors of the rough surface. The rough surface may be represented by a Fourier series with $P_{\vec{K}_{1}}$ and $P_{\vec{K}_{2}}$ being the Fourier coefficients associated with $\vec{K}_{1}$ and $\vec{K}_{2}$, respectively. Here, $\vec{K}=\vec{K}_{1}+\vec{K}_{2}$. Again, with reference to Figure 1, defining $\vec{K}_{s}\left(\widehat{\rho}_{2}, \vec{K}_{1}\right)=k \hat{\rho}_{2}-\vec{K}_{1}$ allows $\chi$ to be expressed as [12]

$$
\chi=j\left(\vec{K}_{1} \cdot \widehat{\rho}_{2}\right)\left(\widehat{K}_{s} \cdot \vec{K}_{2}\right) \cdot G\left[K_{s}\left(\widehat{\rho}_{2}, \vec{K}_{1}\right)\right],
$$

where

$$
G\left[K_{s}\left(\hat{\rho}_{2}, \vec{K}_{1}\right)\right]=\frac{1}{K_{s}}\left\{1-j \frac{k(1+\Delta)}{\sqrt{K_{s}^{2}-k^{2}}+j k \Delta}\right\},
$$

with $\Delta$ being the intrinsic impedance of the surface. At this stage, the current waveform on the dipole source has not been specified. 
2.2. Applications to an FMCW Radar. Following a similar analysis as in [3, 11], (1) may be inversely Fourier-transformed to give the received electric field in the time domain as

$$
\begin{aligned}
& \mathscr{F}^{-1}\left[\left(E_{n}\right)_{1}\right](t)=\frac{1}{(2 \pi)^{3 / 2}} \\
& \cdot \mathscr{F}^{-1}\left[-j \frac{\eta_{0} \Delta l}{c^{2}} \omega^{2} I(\omega)\right] * \mathscr{F}^{-1}\left\{\sum_{\vec{K}} P_{\vec{K}}\right. \\
& \cdot \sqrt{K} e^{j(\rho K / 2) \cos \left(\theta_{K}-\theta\right)} \\
& \cdot \int_{\rho / 2}^{\infty} \frac{F\left(\rho_{1}\right) F\left(\rho_{2}\right)}{\sqrt{\rho_{s}\left[\rho_{s}^{2}-(\rho / 2)^{2}\right]}} e^{-j(\pi / 4)} \sqrt{\cos \phi} \\
& \cdot e^{j k \delta \rho_{0}\left[\cos \phi \cos \left(\theta_{K}-\theta_{0}\right)+\sin \phi \sin \left(\theta_{K}-\theta_{0}\right)\right]} \\
& \left.\cdot e^{j \rho_{s}[K \cos \phi-2 k]} d \rho_{s}\right\} .
\end{aligned}
$$

The current waveform of an FMCW radar may be written as $[6,9]$

$$
i(t)=I_{0} e^{j\left(\omega_{0} t+\alpha \pi t^{2}\right)}\left\{h\left[t+\frac{T_{r}}{2}\right]-h\left[t-\frac{T_{r}}{2}\right]\right\},
$$

where $I_{0}$ is the peak current and $\omega_{0}=2 \pi f_{0}$ is the center radian frequency of the sweep waveform. $T_{r}$ represents the sweep interval and the sweep rate may be expressed as $\alpha=B / T_{r}$ where $B$ is the sweep bandwidth. $h$ is the Heaviside function.

It is known from [9] that, for an FMCW waveform,

$$
\begin{aligned}
\mathscr{F}^{-1} & {\left[-j \frac{\eta_{0} \Delta l}{c^{2}} \omega^{2} I(\omega)\right] } \\
= & -j I_{0} \frac{\eta_{0} \Delta l \omega_{0}^{2}}{c^{2}} e^{j\left(\omega_{0} t+\alpha \pi t^{2}\right)} \\
& \cdot\left\{h\left[t+\frac{T_{r}}{2}\right]-h\left[t-\frac{T_{r}}{2}\right]\right\} .
\end{aligned}
$$

By direct comparison with the corresponding first-order case for a pulsed dipole [11], the first-order time domain electric field for an FMCW source may be written as

$$
\begin{aligned}
& \left(E_{n}\right)_{1}\left(t_{r}\right)=\frac{-j I_{0} \eta_{0} \Delta l k_{0}^{2}}{(2 \pi)^{3 / 2}} \sum_{\vec{K}} P_{\vec{K}} \sqrt{K} e^{j(\rho K / 2) \cos \left(\theta_{K}-\theta\right)} \\
& \cdot \int_{\rho / 2}^{\infty} \frac{F\left(\rho_{1}, \omega_{0}\right) F\left(\rho_{2}, \omega_{0}\right)}{\sqrt{\rho_{s}\left[\rho_{s}^{2}-(\rho / 2)^{2}\right]}} e^{-j(\pi / 4)} \sqrt{\cos \phi} e^{j \rho_{s} K \cos \phi} \\
& \cdot e^{j\left(\omega_{0} t_{r}+\alpha \pi t_{r}^{2}\right)} e^{-j k_{0}\left(2 \rho_{s}-\delta \rho_{s 0}\right)} e^{-j\left(2 \pi \alpha\left(2 \rho_{s}-\delta \rho_{s 0}\right) / c\right) t_{r}} \\
& \cdot e^{j\left(\pi \alpha\left(2 \rho_{s}-\delta \rho_{s 0}\right)^{2} / c^{2}\right)}\left\{h\left[t_{r}+\frac{T_{r}}{2}-\frac{2 \rho_{s}-\delta \rho_{s 0}}{c}\right]\right. \\
& \left.-h\left[t_{r}-\frac{T_{r}}{2}-\frac{2 \rho_{s}-\delta \rho_{s 0}}{c}\right]\right\} d \rho_{s},
\end{aligned}
$$

where $\delta \rho_{s 0}=\delta \rho_{0}\left[\cos \phi \cos \left(\theta_{K}-\theta_{0}\right)+\sin \phi \sin \left(\theta_{K}-\theta_{0}\right)\right] . t$ is renamed as $t_{r}$ to indicate that the time is within a sweep repetition interval $\left(\left(2 \rho_{s}-\delta \rho_{s 0}\right) / c-T_{r} / 2,\left(2 \rho_{s}-\delta \rho_{s 0}\right) / c+\right.$ $\left.T_{r} / 2\right)$. As stated in $[6,9]$, the frequency difference between the transmitted waveform and the received waveform may be Fourier-transformed within this interval to obtain the range information. This is the so-called "range transform." Because the received signals in the given time interval reflect the information for an extremely large range of ocean surface, here range transformation is taken to accurately acquire a patch of ocean surface to analyse. The frequency difference may be obtained by the demodulation process, in which the transmitted signals and the received signals are mixed and then low-pass-filtered.

After the demodulation preprocess, the exponential factor $e^{j\left(\omega_{0} t_{r}+\alpha \pi t_{r}^{2}\right)}$ in (8) will be eliminated. Then, Fouriertransforming with respect to $t_{r}$ gives

$$
\begin{aligned}
\left(E_{n}\right)_{1}\left(\omega_{r}\right) & \\
= & \frac{-j I_{0} \eta_{0} \Delta l k_{0}^{2} T_{r}}{(2 \pi)^{3 / 2}} \sum_{\vec{K}} P_{\vec{K}} \sqrt{K} e^{j(\rho K / 2) \cos \left(\theta_{K}-\theta\right)} \\
& \cdot \int_{\rho / 2}^{\infty} \frac{F\left(\rho_{1}, \omega_{0}\right) F\left(\rho_{2}, \omega_{0}\right)}{\sqrt{\rho_{s}\left[\rho_{s}^{2}-(\rho / 2)^{2}\right]}} e^{-j(\pi / 4)} \sqrt{\cos \phi} e^{j \rho_{s} K \cos \phi} \\
\cdot & e^{-j k_{0}\left(2 \rho_{s}-\delta \rho_{s 0}\right)} e^{j \omega_{r}\left(2 \rho_{s}-\delta \rho_{s 0}\right) / c} e^{-j\left(\pi \alpha\left(2 \rho_{s}-\delta \rho_{s 0}\right)^{2} / c^{2}\right)} \\
\cdot & \operatorname{Sa}\left[\frac{T_{r}}{2}\left(\omega_{r}-\frac{2 \pi \alpha\left(2 \rho_{s}-\delta \rho_{s 0}\right)}{c}\right)\right] d \rho_{s},
\end{aligned}
$$

where $\omega_{r}$ is the transform variable in the frequency domain. Here, it is helpful to define

$$
\rho_{s}^{\prime}=\rho_{s}-\frac{\delta \rho_{s 0}}{2}
$$

Changing the integration variable from $\rho_{s}$ to $\rho_{s}^{\prime}$ and ignoring the $\delta \rho_{s 0} / 2$ factor in the magnitude terms give

$$
\begin{aligned}
\left(E_{n}\right)_{1}\left(\omega_{r}\right) & \\
= & \frac{-j I_{0} \eta_{0} \Delta l k_{0}^{2} T_{r}}{(2 \pi)^{3 / 2}} \sum_{\vec{K}} P_{\vec{K}} \sqrt{K} e^{j(\rho K / 2) \cos \left(\theta_{K}-\theta\right)} \\
& \cdot \int_{\rho / 2}^{\infty} \frac{F\left(\rho_{1}, \omega_{0}\right) F\left(\rho_{2}, \omega_{0}\right)}{\sqrt{\rho_{s}\left[\rho_{s}^{2}-(\rho / 2)^{2}\right]}} e^{-j(\pi / 4)} \sqrt{\cos \phi} \\
\cdot & e^{j\left(K \cos \phi-2 k_{0}+2 \omega_{r} / c\right) \rho_{s}^{\prime}} e^{-j\left(4 \pi \alpha\left(\rho_{s}^{\prime}\right)^{2} / c^{2}\right)} e^{j \delta \rho_{s 0} K \cos \phi / 2} \\
\cdot & \operatorname{Sa}\left[\frac{T_{r}}{2}\left(\omega_{r}-\frac{4 \pi \alpha}{c} \rho_{s}^{\prime}\right)\right] d \rho_{s}^{\prime} .
\end{aligned}
$$

Since the maximum of the sampling function $\mathrm{Sa}(x)$ occurs at $x=0$, a representative range $\rho_{r}$ may be defined as

$$
\rho_{r}=\frac{c \omega_{r}}{4 \pi \alpha} .
$$


Based on the representative range, defining the corresponding range variable

$$
\rho_{s}^{\prime \prime}=\rho_{s}^{\prime}-\rho_{r}
$$

and changing the integration variable from $\rho_{s}^{\prime}$ to $\rho_{s}^{\prime \prime},(11)$ becomes

$$
\begin{aligned}
\left(E_{n}\right)_{1} & \left(\omega_{r}\right) \\
= & \frac{-j I_{0} \eta_{0} \Delta l k_{0}^{2} T_{r}}{(2 \pi)^{3 / 2}} \sum_{\vec{K}} P_{\vec{K}} \sqrt{K} e^{j(\rho K / 2) \cos \left(\theta_{K}-\theta\right)} \\
& \cdot \int_{\rho_{s \min }^{\prime \prime}}^{\rho_{s \max }^{\prime \prime}} \frac{F\left(\rho_{1}, \omega_{0}\right) F\left(\rho_{2}, \omega_{0}\right)}{\sqrt{\rho_{s}\left[\rho_{s}^{2}-(\rho / 2)^{2}\right]}} e^{-j(\pi / 4)} \sqrt{\cos \phi} \\
\cdot & e^{j\left(-2 k_{0}+k_{r}\right) \rho_{r}} e^{j\left(-2 k_{0}\right) \rho_{s}^{\prime \prime}} e^{j \rho_{s} K \cos \phi} \\
& \cdot e^{-j\left(k_{r} / \rho_{r}\right)\left(\rho_{s}^{\prime \prime}\right)^{2}} \mathrm{Sa}\left[k_{B} \rho_{s}^{\prime \prime}\right] d \rho_{s}^{\prime \prime},
\end{aligned}
$$

where $k_{B}=2 \pi B / c$ and $k_{r}=\omega_{r} / c$. A process similar to that in [11] is used to simplify the terms in the integral. Then, (14) reduces to

$$
\begin{aligned}
\left(E_{n}\right)_{1}\left(\omega_{r}\right)= & \frac{-j I_{0} \eta_{0} \Delta l k_{0}^{2} T_{r}}{(2 \pi)^{3 / 2}} \sum_{\vec{K}} P_{\vec{K}} \sqrt{K} e^{j(\rho K / 2) \cos \left(\theta_{K}-\theta\right)} \\
& \cdot e^{-j(\pi / 4)} \sqrt{\cos \phi_{0}} e^{j\left(K \cos \phi_{0}-2 k_{0}+k_{r}\right) \rho_{r}} \\
& \cdot \frac{F\left(\rho_{01}, \omega_{0}\right) F\left(\rho_{02}, \omega_{0}\right)}{\sqrt{\rho_{r}\left[\rho_{r}^{2}-(\rho / 2)^{2}\right]}} e^{j\left(\delta \rho_{s 0} / 2\right)\left(K / \cos \phi_{0}\right)} \\
& \cdot \int_{\rho_{s \min }^{\prime \prime}}^{\rho_{s \max }^{\prime \prime}} e^{j\left(K / \cos \phi_{0}-2 k_{0}\right) \rho_{s}^{\prime \prime}} \\
& \cdot e^{-j\left(k_{r} / \rho_{r}\right)\left(\rho_{s}^{\prime \prime}\right)^{2}} \mathrm{Sa}\left[k_{B} \rho_{s}^{\prime \prime}\right] d \rho_{s}^{\prime \prime},
\end{aligned}
$$

where $\rho_{01}$ and $\rho_{02}$ are representative values of $\rho_{1}$ and $\rho_{2}$ (see Figure 1), respectively. By directly comparing (15) with (24) in [9], the first-order bistatic received electric field for an FMCW waveform with an antenna on a floating platform may be expressed as

$$
\begin{aligned}
& \left(E_{n}\right)_{1}\left(\omega_{r}\right)=\frac{-j I_{0} \eta_{0} \Delta l k_{0}^{2}}{(2 \pi)^{3 / 2}} \sum_{\vec{K}} P_{\vec{K}} \sqrt{K} e^{j(\rho K / 2) \cos \left(\theta_{K}-\theta\right)} \\
& \cdot e^{-j(\pi / 4)} \sqrt{\cos \phi_{0}} e^{j\left(K \cos \phi_{0}-2 k_{0}+k_{r}\right) \rho_{r}} \\
& \cdot \frac{F\left(\rho_{01}, \omega_{0}\right) F\left(\rho_{02}, \omega_{0}\right)}{\sqrt{\rho_{r}\left[\rho_{r}^{2}-(\rho / 2)^{2}\right]}} e^{j\left(\delta \rho_{s 0} / 2\right)\left(K / \cos \phi_{0}\right)} \\
& \text { - }\left(T_{r} \Delta \rho\right) \operatorname{Sm}\left(K, \cos \phi_{0}, k_{B}, \Delta r\right) \text {. }
\end{aligned}
$$

$\pm \Delta r$ are the symmetrical limits of the integral in (15), where a sampling function dominates this integral. If only the values of $\rho_{s}^{\prime \prime}$ within the main lobe of the sampling function are considered in the integral, that is, $-\pi / 2<k_{B} \rho_{s}^{\prime \prime}<\pi / 2$, it can be deduced as in [9] that $\Delta r=\Delta \rho / 2=c / 4 B$. Consider

$$
\begin{aligned}
\operatorname{Sm} & \left(K, \cos \phi_{0}, k_{B}, \Delta r\right) \\
= & \frac{1}{\pi}\left\{\operatorname{Si}\left[\left(\frac{K}{\cos \phi_{0}}-2 k_{0}+k_{B}\right) \Delta r\right]\right. \\
& \left.-\operatorname{Si}\left[\left(\frac{K}{\cos \phi_{0}}-2 k_{0}-k_{B}\right) \Delta r\right]\right\},
\end{aligned}
$$

where $\operatorname{Si}(x)=\int_{0}^{x}(\sin (t) / t) d t$.

Following a similar procedure to the first-order case, the second-order bistatic received electric field with a transmitter on a floating platform for an FMCW waveform may be written as

$$
\begin{aligned}
\left(E_{n}\right)_{2 E}\left(\omega_{r}\right) & \\
= & \frac{-j I_{0} \eta_{0} \Delta l k_{0}^{2}}{(2 \pi)^{3 / 2}} \sum_{\vec{K}_{1} \vec{K}_{2}} P_{\vec{K}_{1}} P_{\vec{K}_{2}} \sqrt{K} e^{-j(\pi / 4)} \\
& \cdot e^{j(\rho K / 2) \cos \left(\theta_{K}-\theta\right)} \sqrt{\cos \phi_{0}} e^{j\left(K \cos \phi_{0}-2 k_{0}+k_{r}\right) \rho_{r}} \\
& \cdot \frac{\mathrm{SE} \Gamma_{P} F\left(\rho_{02}, \omega_{0}\right) F\left(\rho_{020}, \omega_{0}\right)}{\sqrt{\rho_{r}\left[\rho_{r}^{2}-(\rho / 2)^{2}\right]}} e^{j\left(\delta \rho_{s 0} / 2\right)\left(K / \cos \phi_{0}\right)} \\
& \cdot\left(T_{r} \Delta \rho\right) \operatorname{Sm}\left(K, \cos \phi_{0}, k_{B}, \Delta r\right),
\end{aligned}
$$

where $\rho_{02}$ and $\rho_{020}$ are the representative values of $\rho_{2}$ and $\rho_{20}$, respectively. The symmetrical electromagnetic coupling coefficient, ${ }_{\mathrm{SE}} \Gamma_{P}$, may be expressed as [12]

$$
\begin{aligned}
\mathrm{SE} & \Gamma_{P}\left(\vec{K}_{1}, \vec{K}_{2}\right)=\frac{j k_{0}}{2 K \cos \phi_{0}}\left\{\left(\vec{K}_{1} \cdot \widehat{\rho}_{2}\right)\right. \\
& \cdot\left[\widehat{K}_{s}\left(\hat{\rho}_{2}, \vec{K}_{1}\right) \cdot \vec{K}_{2}\right] G\left[K_{s}\left(\widehat{\rho}_{2}, \vec{K}_{1}\right)\right] \\
& \left.+\left(\vec{K}_{2} \cdot \widehat{\rho}_{2}\right)\left[\widehat{K}_{s}\left(\widehat{\rho}_{2}, \vec{K}_{2}\right) \cdot \vec{K}_{1}\right] G\left[K_{s}\left(\hat{\rho}_{2}, \vec{K}_{2}\right)\right]\right\}
\end{aligned}
$$

with $\vec{K}_{s}\left(\hat{\rho}_{2}, \vec{K}_{2}\right)=k \hat{\rho}_{2}-\vec{K}_{2}$ and

$$
G\left[K_{s}\left(\hat{\rho}_{2}, \vec{K}_{1}\right)\right]=\frac{1}{K_{s}}\left\{1-j \frac{k(1+\Delta)}{\sqrt{K_{s}^{2}-k^{2}}+j k \Delta}\right\}
$$




\section{Radar Cross Sections}

3.1. First-Order Radar Cross Section. In developing the ocean radar cross section, a time-varying ocean surface, represented as $\xi(\vec{\rho}, t)=\sum_{\vec{\rho}, t} P_{\vec{K}, \omega} e^{j \vec{K} \cdot \vec{\rho}} e^{j \omega t}$, is used to replace the timeinvariant case $\xi(\vec{\rho})=\sum_{\vec{\rho}} P_{\vec{K}} e^{j \vec{K} \cdot \vec{\rho}}$. This gives the time-varying received electric field corresponding to (16) as

$$
\begin{aligned}
\left(E_{n}\right)_{1}\left(\omega_{r}, t\right) & \\
= & \frac{-j I_{0} \eta_{0} \Delta l k_{0}^{2}}{(2 \pi)^{3 / 2}} \sum_{\vec{K}, \omega} P_{\vec{K}, \omega} \sqrt{K} e^{j(\rho K / 2) \cos \left(\theta_{K}-\theta\right)} \\
& \cdot e^{j\left(K \cos \phi_{0}-2 k_{0}+k_{r}\right) \rho_{r}} e^{-j(\pi / 4)} \sqrt{\cos \phi_{0}} e^{j \omega t} \\
& \cdot \frac{F\left(\rho_{01}, \omega_{0}\right) F\left(\rho_{02}, \omega_{0}\right)}{\sqrt{\rho_{r}\left[\rho_{r}^{2}-(\rho / 2)^{2}\right]}} e^{j\left(\delta \rho_{s 0} / 2\right)\left(K / \cos \phi_{0}\right)} \\
& \cdot\left(T_{r} \Delta \rho\right) \operatorname{Sm}\left(K, \cos \phi_{0}, k_{B}, \Delta r\right) .
\end{aligned}
$$

A technique similar to that in $[9,11]$ is used to obtain the radar cross section from the received electric field equation. The initial step of the approach is to write the autocorrelation, $R(\tau)$, as

$$
R(\tau)=\frac{A_{r}}{2 \eta_{0}} \frac{1}{T_{r}^{2}}\left\langle\left(E_{n}\right)_{1}\left(\omega_{r}, t+\tau\right)\left(E_{n}\right)_{1}^{*}\left(\omega_{r}, t\right)\right\rangle,
$$

where $A_{r}=\left(\lambda_{0}^{2} / 4 \pi\right) G_{r}$, with $G_{r}$ being the gain of the receiving array. Here, $\omega_{r}$ represents the fixed patch over two sweep intervals, whose time interval is $\tau$.

After Fourier-transforming the autocorrelation and comparing directly with the radar range equation, the radar cross section, $\sigma_{1}\left(\omega_{d}\right)$, may be written as

$$
\begin{aligned}
& \sigma_{1}\left(\omega_{d}\right)=2^{3} \pi k_{0}^{2} \Delta \rho \sum_{m= \pm 1} \int_{K} S_{1}(m \vec{K}) K^{2} \cos \phi_{0} \\
& \cdot \operatorname{Sm}^{2}\left(K, \cos \phi_{0}, k_{B}, \Delta r\right) \\
& \cdot\left\{J _ { 0 } ^ { 2 } \left[\frac{a K}{2} \mid \cos \left(\theta_{K}-\theta_{K_{p}}\right)\right.\right. \\
& \left.+\tan \phi_{0} \sin \left(\theta_{K}-\theta_{K_{p}}\right) \mid\right] \cdot \delta\left(\omega_{d}+m \sqrt{g K}\right) \\
& +\sum_{n=1}^{\infty} J_{n}^{2}\left[\frac{a K}{2} \mid \cos \left(\theta_{K}-\theta_{K_{p}}\right)\right. \\
& \left.+\tan \phi_{0} \sin \left(\theta_{K}-\theta_{K_{p}}\right) \mid\right] \cdot\left[\delta \left(\omega_{d}+m \sqrt{g K}\right.\right. \\
& \left.\left.\left.+n \omega_{p}\right)+\delta\left(\omega_{d}+m \sqrt{g K}+n \omega_{p}\right)\right]\right\} d K,
\end{aligned}
$$

where $J_{n}$ represents the $n$ th-order Bessel functions. For simulation purposes (see Section 4) and in keeping with [4, 11], it will be assumed that the antenna motion is caused by the dominant ocean waves. $a, \omega_{p}$, and $\theta_{K_{p}}$ represent the antenna platform sway amplitude, frequency, and direction, respectively.

3.2. Second-Order Radar Cross Section. It is known that the second-order radar cross section contains two portions: an hydrodynamic contribution and an electromagnetic contribution. Using the Fourier coefficient for the second-order

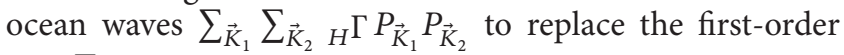
case $\sum_{\vec{K}} P_{\vec{K}}$ in (16), the hydrodynamic second-order electric field may be written as

$$
\begin{aligned}
\left(E_{n}\right)_{2 H}\left(\omega_{r}\right) & \\
= & \frac{-j I_{0} \eta_{0} \Delta l k_{0}^{2}}{(2 \pi)^{3 / 2}} \sum_{\vec{K}_{1}} \sum_{\vec{K}_{2}} P_{\vec{K}_{1}} P_{\vec{K}_{2}} \sqrt{K} e^{-j(\pi / 4)} \\
& \cdot e^{j(\rho K / 2) \cos \left(\theta_{K}-\theta\right)} \sqrt{\cos \phi_{0}} e^{j\left(K \cos \phi_{0}-2 k_{0}+k_{r}\right) \rho_{r}} \\
& \cdot \frac{{ }_{H} \Gamma F\left(\rho_{01}, \omega_{0}\right) F\left(\rho_{02}, \omega_{0}\right)}{\sqrt{\rho_{r}\left[\rho_{r}^{2}-(\rho / 2)^{2}\right]}} e^{j\left(\delta \rho_{s 0} / 2\right)\left(K / \cos \phi_{0}\right)} \\
& \cdot\left(T_{r} \Delta \rho\right) \operatorname{Sm}\left(K, \cos \phi_{0}, k_{B}, \Delta r\right)
\end{aligned}
$$

where ${ }_{H} \Gamma$ is the hydrodynamic coupling coefficient [13], accounting for the coupling of two first-order ocean waves, whose wavenumbers are $K_{1}$ and $K_{2}$, respectively. Adding the electromagnetic contribution (18) and the hydrodynamic contribution (24) together and using the time-varying ocean wave surface to replace the time-invariant case, the total second-order bistatic electric field for an FMCW source with an antenna on a floating platform may be expressed as

$$
\begin{aligned}
\left(E_{n}\right)_{2} & \left(\omega_{r}, t\right) \\
= & \frac{-j I_{0} \eta_{0} \Delta l k_{0}^{2}}{(2 \pi)^{3 / 2}} \sum_{\vec{K}_{1}, \omega_{1}} \sum_{\vec{K}_{2}, \omega_{2}} P_{\vec{K}_{1}, \omega_{1}} P_{\vec{K}_{2}, \omega_{2}} \\
& \cdot{ }_{S} \Gamma_{P} \sqrt{K} e^{j(\rho K / 2) \cos \left(\theta_{K}-\theta\right)} e^{-j(\pi / 4)} \\
& \cdot \sqrt{\cos \phi_{0}} e^{j\left(K \cos \phi_{0}-2 k_{0}+k_{r}\right) \rho_{r}} e^{j \omega t} \\
& \cdot \frac{F\left(\rho_{01}, \omega_{0}\right) F\left(\rho_{02}, \omega_{0}\right)}{\sqrt{\rho_{r}\left[\rho_{r}^{2}-(\rho / 2)^{2}\right]}} e^{j\left(\delta \rho_{s 0} / 2\right)\left(K / \cos \phi_{0}\right)} \\
& \cdot\left(T_{r} \Delta \rho\right) \operatorname{Sm}\left(K, \cos \phi_{0}, k_{B}, \Delta r\right),
\end{aligned}
$$

where ${ }_{S} \Gamma_{P}={ }_{\mathrm{SE}} \Gamma_{P}+{ }_{H} \Gamma$.

Following the same procedure as for the first-order case, based on the total second-order time-varying received electric field (25), the corresponding second-order radar cross section, $\sigma_{2}\left(\omega_{d}\right)$, may be obtained as 


$$
\begin{aligned}
\sigma_{2}\left(\omega_{d}\right)=2^{3} \pi k_{0}^{2} \Delta \rho \sum_{m_{1}= \pm 1} \sum_{m_{2}= \pm 1} \int_{0}^{\infty} \int_{-\pi}^{\pi} \int_{0}^{\infty} S_{1}\left(m_{1} \vec{K}_{1}\right) S_{1}\left(m_{2} \vec{K}_{2}\right)\left|{ }_{S} \Gamma_{P}\right|^{2} K^{2} \cos \phi_{0} K_{1} \operatorname{sm}^{2}\left(K, \cos \phi_{0}, k_{B}, \Delta r\right) \\
.\left\{J_{0}^{2}\left\{\frac{a K}{2}\left|\cos \left(\theta_{K}-\theta_{K_{p}}\right)+\tan \phi_{0} \sin \left(\theta_{K}-\theta_{K_{p}}\right)\right|\right\} \cdot \delta\left(\omega_{d}+m_{1} \sqrt{g K_{1}}+m_{2} \sqrt{g K_{2}}\right)\right. \\
+\sum_{n=1}^{\infty} J_{n}^{2}\left\{\frac{a K}{2}\left|\cos \left(\theta_{K}-\theta_{K_{p}}\right)+\tan \phi_{0} \sin \left(\theta_{K}-\theta_{K_{p}}\right)\right|\right\} \\
\left.\cdot\left[\delta\left(\omega_{d}+m_{1} \sqrt{g K_{1}}+m_{2} \sqrt{g K_{2}}-n \omega_{p}\right)+\delta\left(\omega_{d}+m_{1} \sqrt{g K_{1}}+m_{2} \sqrt{g K_{2}}+n \omega_{p}\right)\right]\right\} d K_{1} d \theta_{\vec{K}_{1}} d K .
\end{aligned}
$$

\section{Simulation and Discussion}

Based on a Pierson-Moskowitz (PM) ocean spectral model [14], the newly derived radar cross sections, accounting for antenna sway, can be simulated to illustrate the differences in the FMCW and pulsed waveform cases. The sweep bandwidth of the FMCW waveform is chosen as $50 \mathrm{kHz}$. The operating frequency, defined as the central frequency of the FMCW waveform, is taken to be $25 \mathrm{MHz}$. The bistatic angle is $30^{\circ}$ and the wind speed is 20 knots. The scattering ellipse normal and the wind direction are $90^{\circ}$ and $180^{\circ}$, respectively, as measured from the positive $x$-axis (the line connecting the transmitter with the receiver). The sway amplitude and frequency depend on the wind velocity, and in keeping with an example used earlier [4], for the purpose of illustration, here these values are taken as $1.228 \mathrm{~m}$ and $0.127 \mathrm{~Hz}$, respectively. The sway direction is chosen to be the same as the wind direction.

4.1. First-Order Radar Cross Section. Figure 2 shows a comparison of the first-order radar cross section for a pulsed source and that for an FMCW source. For the FMCW waveform, $\Delta r=1500 \mathrm{~m}$, which equals half the width of the scattering patch $(\Delta \rho=3000 \mathrm{~m})$ for the pulsed waveform, in order to keep the same bandwidth for both waveforms. A hamming window is used to smooth the curve and reduce the oscillations. From this figure, it can be observed that additional peaks caused by the antenna motion appear symmetrically in the Doppler spectrum with respect to the Bragg peaks. A detailed description and properties of these motion-induced peaks were discussed in [11]. It can also be seen that the magnitude of the radar cross sections for the FMCW waveform is a little lower than that for the corresponding pulsed waveform, which may be caused by the value of $\Delta r . \Delta r$ is the limit value of the integral, where a sampling function dominates this integral. $\Delta r$ is usually taken to be $\Delta r=\Delta \rho / 2$, which means only the contributions in the main lobe of the sampling function are considered and no interaction between the range bins is assumed in the ideal case.

It is clear that the first-order radar cross section has a certain relationship with the integral limit $\Delta r$. In Section 2, it may be observed that there is no mathematical limit for the parameter $\Delta r$. By varying $\Delta r$, the effect on the radar cross section can be examined. Keeping the value of $\Delta \rho=3000 \mathrm{~m}$,
$\Delta r=0.5 \Delta \rho$ and $\Delta r=10 \Delta \rho$ are simulated in Figures 3(a) and 3 (b), respectively. It should be mentioned that the hamming window smoothing process is not used in Figure 3 in order to clearly show the side lobe levels of the first-order radar cross sections. The side lobe structure appears in the radar Doppler spectra due to the side lobes of the Sm function for the FMCW waveform. By comparing Figures 3(a) and 3(b), the magnitude of the side lobes for FMCW source is found to decrease with increasing $\Delta r$ and the main lobe level is a little raised with increasing $\Delta r$ due to the properties of the Sm function. This seems to indicate an advantage of an FMCW system. When the value of $\Delta r$ is taken to be larger than $\Delta \rho / 2$, the interactions between the range bins (the contributions in the side lobe of the sampling function) are considered and appear in the received electric field at a fixed distance. Increasing $\Delta r$ means the received signal is scattered from a larger ocean surface region. When $\Delta r$ approaches infinity, the radar cross section for the FMCW waveform becomes a rectangular function, whose width is determined by $B /\left(2 f_{0} \omega_{B}\right)$. However, when the patch width $\Delta \rho$ approaches infinity, the sampling functions in the first-order pulse radar ocean cross section reduce to delta functions.

By varying the radar bandwidth and keeping the relationships $\Delta \rho=c / 2 B$ and $\Delta r=\Delta \rho / 2$, the effect of the bandwidth on the radar cross sections is illustrated in Figure 4. From this figure, it can be seen that, with increased bandwidth, the magnitudes of the Bragg peaks and the motion-induced peaks are found to be reduced, while the rest of the radar cross section increases. In addition, the width of the Bragg peaks and the motion-induced peaks is also broadened. Therefore, if a large radar bandwidth is used for ocean remote sensing, the Bragg peaks may be significantly contaminated by the motion-induced peaks.

4.2. Second-Order Radar Cross Section. A similar technique is used to simplify and simulate the second-order radar ocean cross section for the FMCW waveform as that for the pulsed waveform in $[12,15]$. For the case of large $\Delta r$, it can be shown that

$$
\begin{aligned}
\lim _{\Delta r \rightarrow \infty} & {\left[\Delta \rho \operatorname{Sm}^{2}\left(K, \cos \phi_{0}, k_{B}, \Delta r\right)\right] \approx \Delta \rho \cos \phi_{0} } \\
\cdot & \left\{h\left[K-\cos \phi_{0}\left(2 k_{0}-k_{B}\right)\right]\right. \\
& \left.-h\left[K-\cos \phi_{0}\left(2 k_{0}+k_{B}\right)\right]\right\} .
\end{aligned}
$$




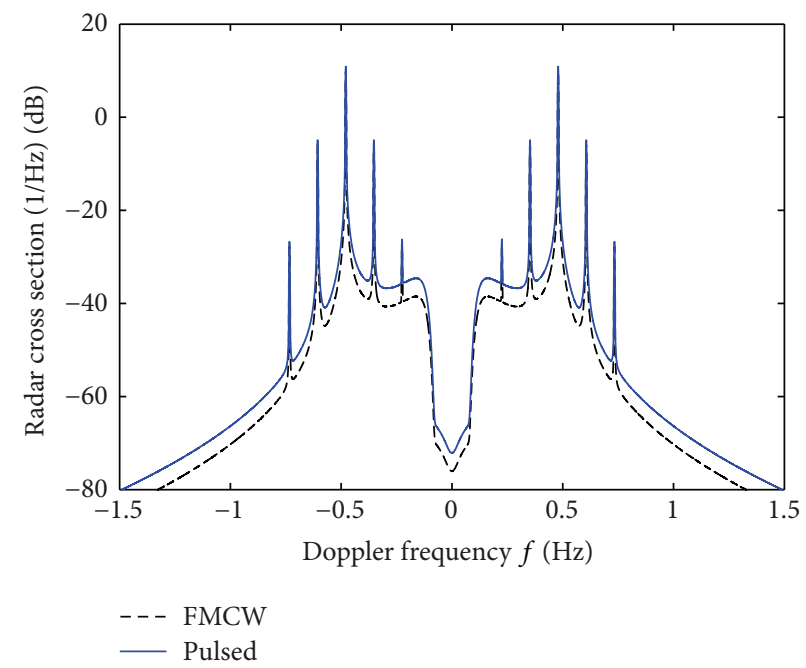

Figure 2: Comparison of the first-order radar cross sections for the FMCW waveform with that for the pulsed waveform.

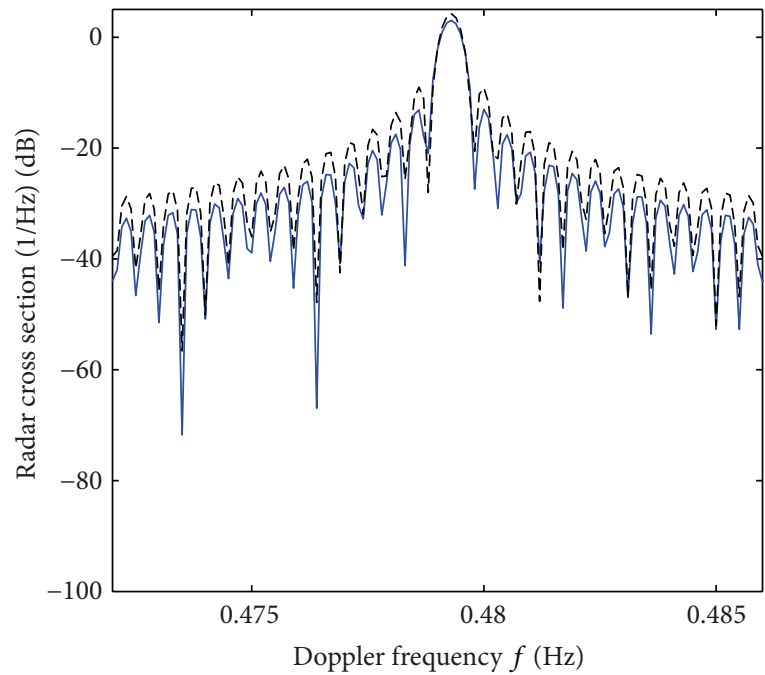

- FMCW

- . - Pulsed

(a)

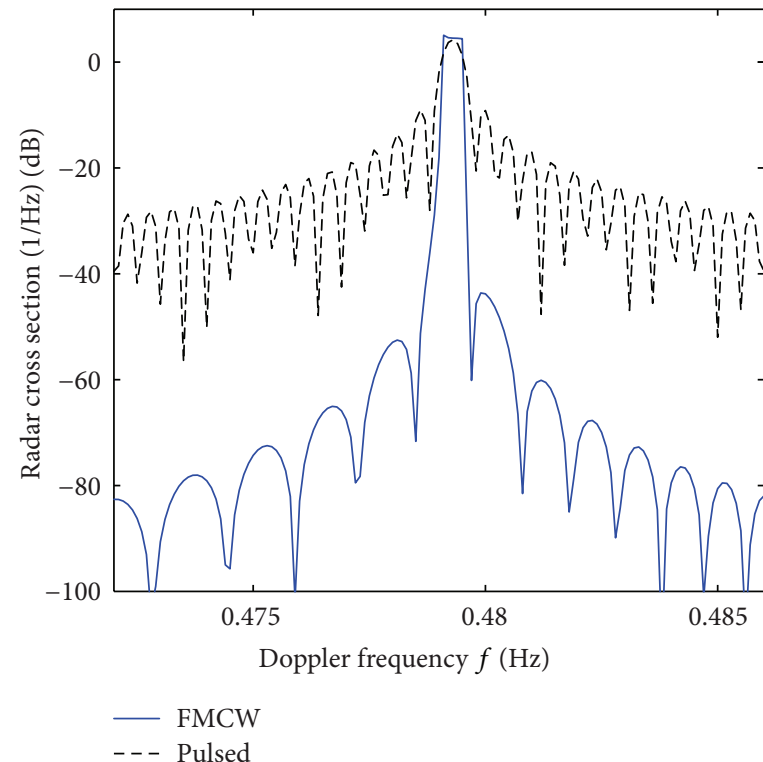

(b)

FIGURE 3: Comparison of the side lobe levels of the first-order radar cross sections for the pulsed and FMCW waveform. (a) $\Delta r=0.5 \Delta \rho$ and (b) $\Delta r=10 \Delta \rho$.

Assuming that the other terms in (26) are slowly varying within the interval

$$
\cos \phi_{0}\left(2 k_{0}-k_{B}\right)<K<\cos \phi_{0}\left(2 k_{0}+k_{B}\right)
$$

and carrying out the $K$ integration, (26) reduces to

$$
\begin{aligned}
\sigma_{2}\left(\omega_{d}\right) & =64 \pi^{2} k_{0}^{4} \cos ^{4} \phi_{0} \sum_{m_{1}= \pm 1} \sum_{m_{2}= \pm 1} \int_{0}^{\infty} \int_{-\pi}^{\pi} S_{1}\left(m_{1} \vec{K}_{1}\right) \\
\cdot & S_{1}\left(m_{2} \vec{K}_{2}\right)\left|{ }_{S} \Gamma_{P}\right|^{2} K_{1} \\
\cdot & \left\{J _ { 0 } ^ { 2 } \left\{\frac{a K}{2} \mid \cos \left(\theta_{K}-\theta_{K_{p}}\right)+\tan \phi_{0}\right.\right. \\
\cdot & \left.\sin \left(\theta_{K}-\theta_{K_{p}}\right) \mid\right\}
\end{aligned}
$$




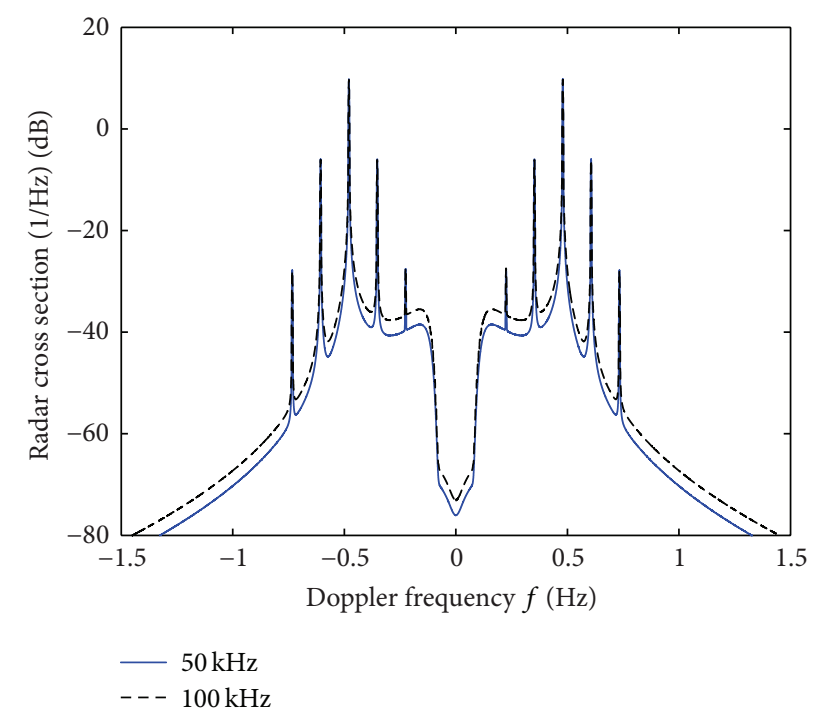

FIGURE 4: The effect of the bandwidth on the first-order radar cross sections.

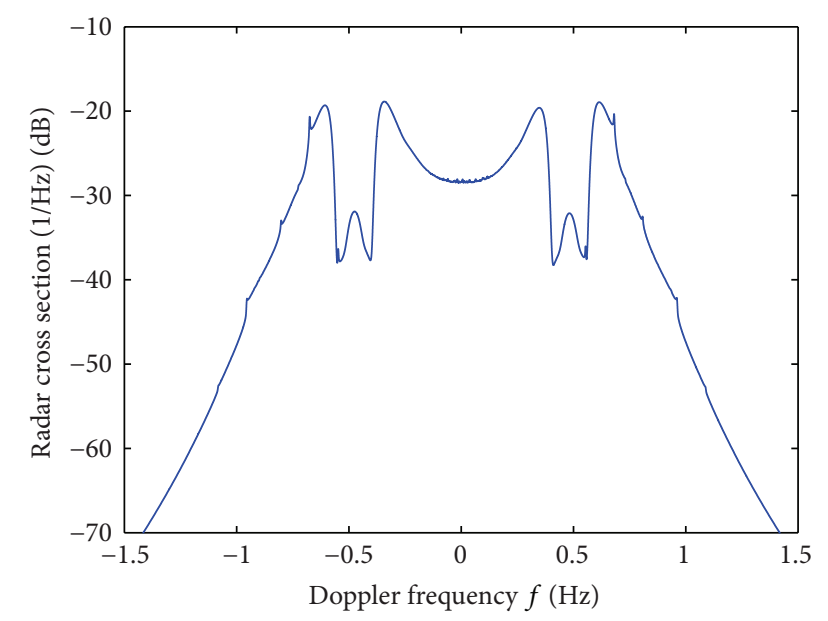

FIGURE 5: Second-order bistatic radar cross section with a transmitter on a floating platform.

Equation (29) is exactly the same as the corresponding model for the pulsed waveform when the scattering patch $\Delta \rho$ approaches infinity. Therefore, the second-order cross section model for the FMCW waveform shows the same features in the Doppler spectra as the model for the pulsed waveform in [12], for a given sea state, radar operating parameters, and platform motion. An example of the second-order bistatic radar cross section with a transmitter on a floating platform and a fixed receiver is shown in Figure 5, for the case of the scattering patch being assumed to be infinite in extent.

\section{Conclusion}

The first- and second-order bistatic radar ocean cross sections for an antenna on a floating platform have been presented for the case of an FMCW waveform. In the derivation process, the first- and second-order models begin with the bistatically received electric field equations derived in $[11,12]$. Subsequently, the derivation is carried out for an FMCW radar, which is different from [11, 12] where a pulsed radar is considered. In particular, the distinguishing feature between the current work and that presented earlier is that demodulation and range transformation must be used to obtain the range information. Based on the new models, simulations are made to compare the radar cross sections for the FMCW waveform with that for the pulsed waveform. It is found that the first-order radar cross section for the FMCW waveform is a little lower than that for a pulsed source with the same simulation parameters. With increased radar operating bandwidth, the magnitude and width of Bragg peaks and motion-induced peaks are found to be reduced and broadened, respectively. For an FMCW waveform, there is no definite mathematical limit for a patch width, which is different from that for a pulsed waveform. Therefore, the magnitude of the range bin is varied to examine the effect on the radar cross section. The side lobe level is found to be reduced with increasing magnitude of the range bin. When the range bin approaches infinity, the first-order radar cross section for an FMCW waveform approaches a rectangular function and the second-order radar cross section model for the FMCW waveform is reduced to that of the pulsed waveform.

\section{Competing Interests}

The authors declare that there are no competing interests regarding the publication of this paper.

\section{Acknowledgments}

The work was supported in part by Natural Sciences and Engineering Research Council of Canada (NSERC) under Discovery Grants to Weimin Huang (NSERC 402313-2012) and Eric W. Gill (NSERC 238263-2010 and RGPIN-201505289) and by an Atlantic Innovation Fund Award (Eric W. Gill, principal investigator).

\section{References}

[1] D. E. Barrick, "First-order theory and analysis of MF/HF/VHF scatter from the sea," IEEE Transactions on Antennas and Propagation, vol. 20, no. 1, pp. 2-10, 1972.

[2] J. Walsh and E. W. Gill, "An analysis of the scattering of highfrequency electromagnetic radiation from rough surfaces with application to pulse radar operating in backscatter mode," Radio Science, vol. 35, no. 6, pp. 1337-1359, 2000.

[3] E. W. Gill and J. Walsh, "High-frequency bistatic cross sections of the ocean surface," Radio Science, vol. 36, no. 6, pp. 1459-1475, 2001.

[4] J. Walsh, W. Huang, and E. Gill, “The first-order high frequency radar ocean surface cross section for an antenna on a floating platform," IEEE Transactions on Antennas and Propagation, vol. 58, no. 9, pp. 2994-3003, 2010.

[5] J. Walsh, W. Huang, and E. Gill, “The second-order high frequency radar ocean surface cross section for an antenna 
on a floating platform," IEEE Transactions on Antennas and Propagation, vol. 60, no. 10, pp. 4804-4813, 2012.

[6] D. E. Barrick, "FM/CW radar signals and digital processing," Tech. Rep. ERL 283-WPL 26, NOAA, 1973.

[7] R. Khan, B. Gamberg, D. Power et al., "Target detection and tracking with a high frequency ground wave radar," IEEE Journal of Oceanic Engineering, vol. 19, no. 4, pp. 540-548, 1994.

[8] A. Wojthiewicz, J. Misiurewicz, M. Nalecz, K. Jedrzejewski, and K. Kulpa, "Two dimensional signal processing in FMCW radars," in Proceedings of the Conference on Circuit Theory and Electronics Circuits, pp. 474-480, Kolobrzeg, Poland, October 1997.

[9] J. Walsh, J. Zhang, and E. W. Gill, "High-frequency radar cross section of the ocean surface for an FMCW waveform," IEEE Journal of Oceanic Engineering, vol. 36, no. 4, pp. 615-626, 2011.

[10] S. Chen, E. W. Gill, and W. Huang, "A first-order HF radar crosssection model for mixed-path ionosphere-ocean propagation with an FMCW source," IEEE Journal of Oceanic Engineering, 2016.

[11] Y. Ma, E. Gill, and W. Huang, "The first-order bistatic high frequency radar ocean surface cross section for an antenna on a floating platform," IET Radar, Sonar \& Navigation, vol. 10, no. 6, pp. 1136-1144, 2016.

[12] Y. Ma, W. Huang, and E. Gill, "The second-order bistatic high frequency radar ocean surface cross section for an antenna on a floating platform," Canadian Journal of Remote Sensing, vol. 42, no. 4, pp. 332-343, 2016.

[13] K. Hasselmann, "On the non-linear energy transfer in a gravitywave spectrum part 1. General theory," Journal of Fluid Mechanics, vol. 12, pp. 481-500, 1962.

[14] W. J. Pierson and L. Moskowitz, "A proposed spectral form for fully developed wind seas based on the similarity theory of S. A. Kitaigorodskii," Journal of Geophysical Research, vol. 69, no. 24, pp. 5181-5190, 1964.

[15] B. J. Lipa and D. E. Barrick, "Extraction of sea state from HF radar sea echo: mathematical theory and modeling," Radio Science, vol. 21, no. 1, pp. 81-100, 1986. 


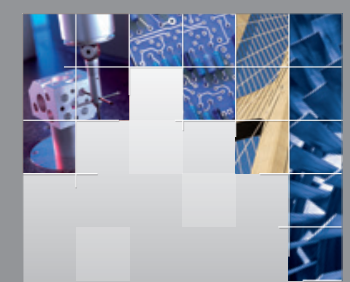

\section{Enfincering}
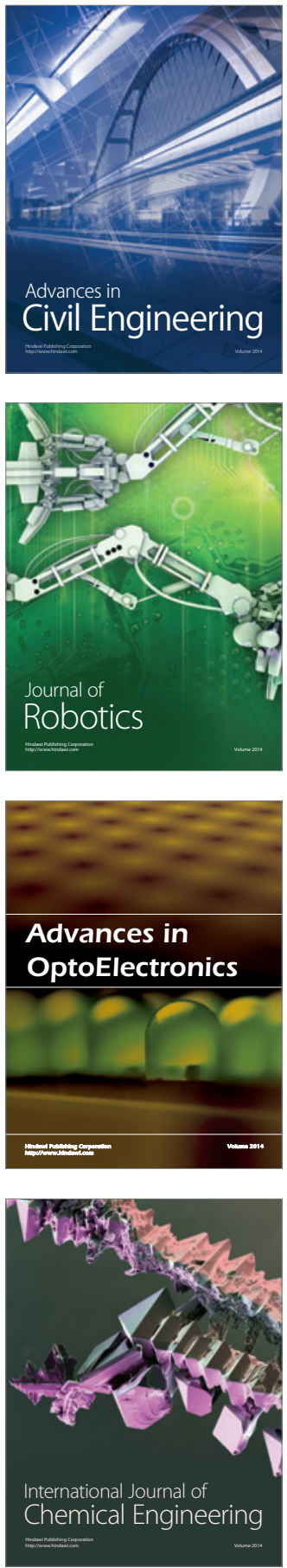

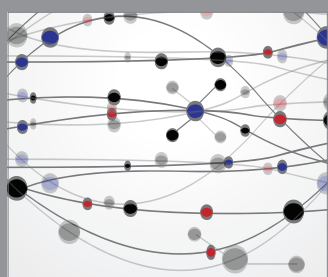

The Scientific World Journal

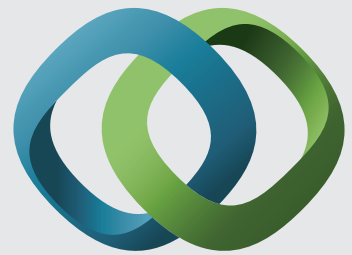

\section{Hindawi}

Submit your manuscripts at

http://www.hindawi.com
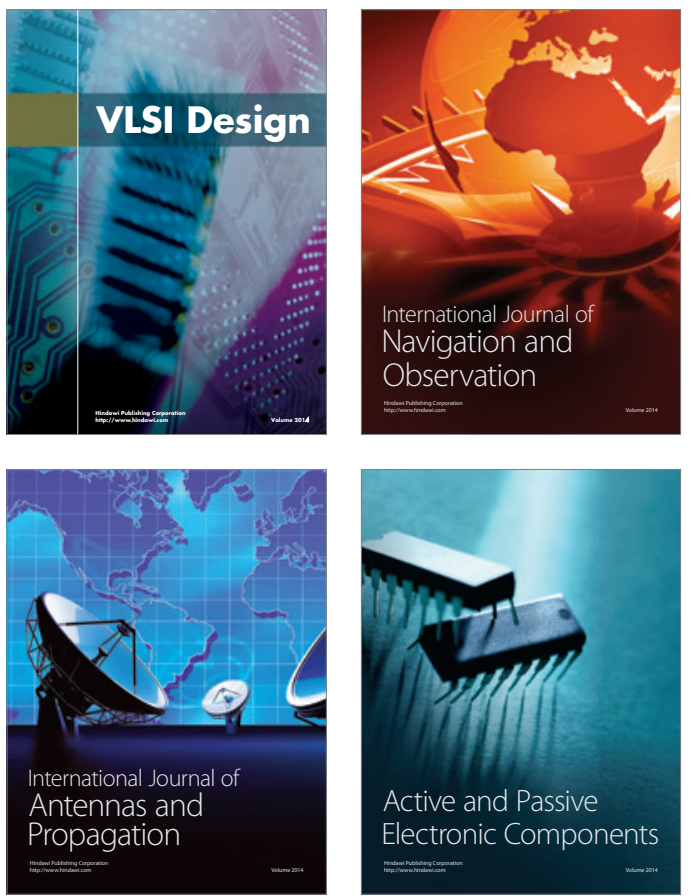
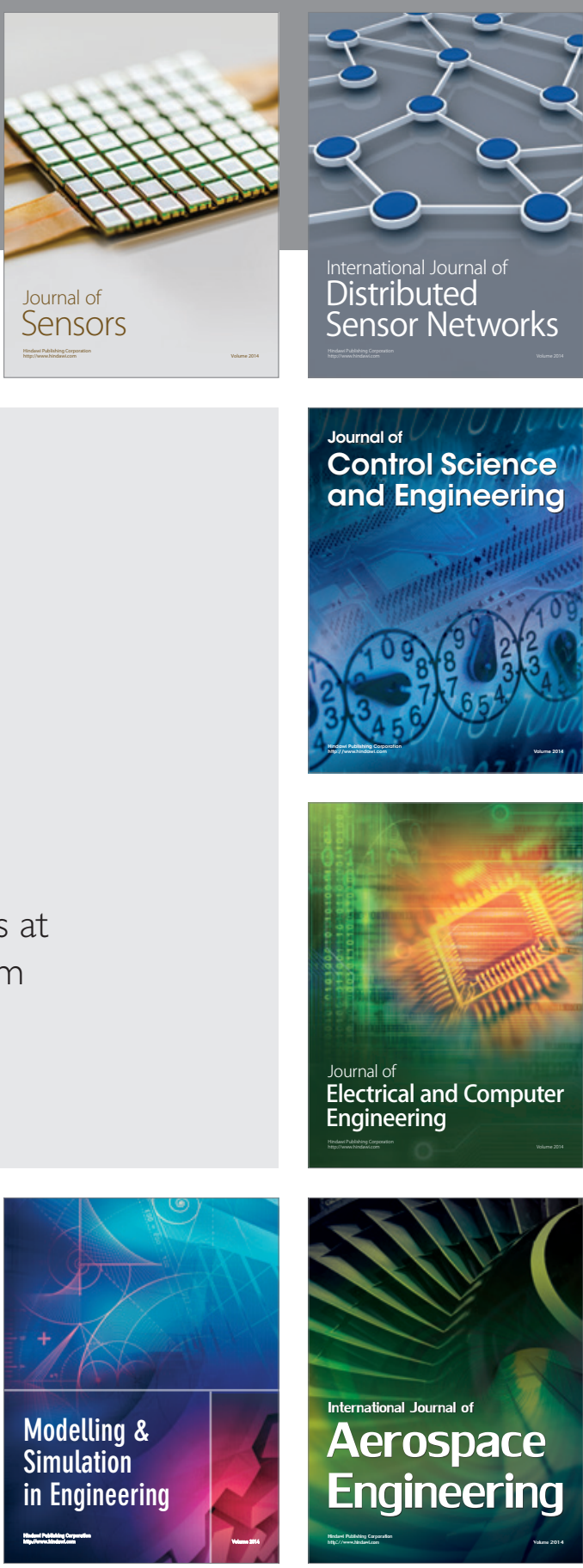

International Journal of

Distributed

Sensor Networks

Journal of

Control Science

and Engineering
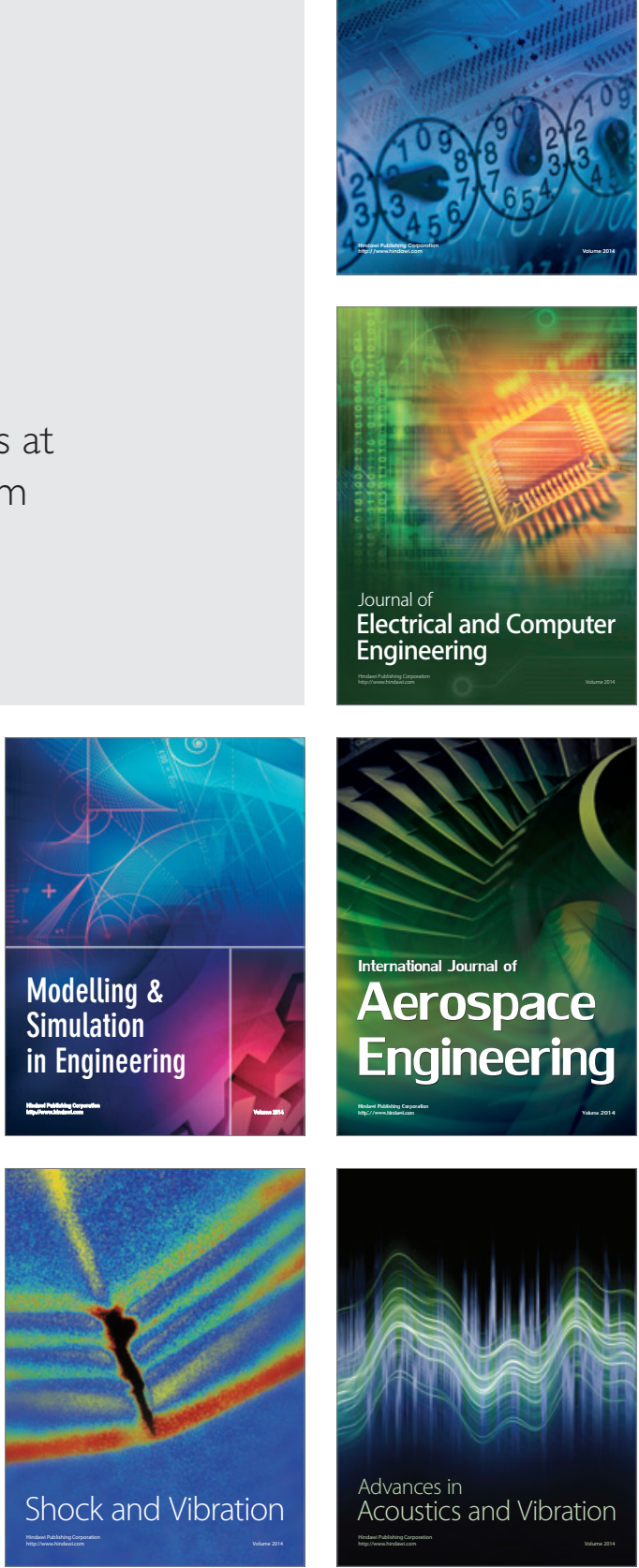